University of New Hampshire

University of New Hampshire Scholars' Repository

Space Science Center

Institute for the Study of Earth, Oceans, and

Space (EOS)

$7-29-2010$

\title{
Plans for the first balloon flight of the gamma-ray polarimeter experiment (GRAPE)
}

\author{
Taylor Connor \\ University of New Hampshire - Manchester \\ Chris Bancroft \\ University of New Hampshire \\ Peter F. Bloser \\ University of New Hampshire, Peter.Bloser@unh.edu \\ Jason S. Legere \\ University of New Hampshire, jslegere@unh.edu \\ Mark L. McConnell \\ University of New Hampshire - Main Campus, mark.mcconnell@unh.edu
}

See next page for additional authors

Follow this and additional works at: https://scholars.unh.edu/ssc

Part of the Astrophysics and Astronomy Commons

\section{Recommended Citation}

Taylor P. Connor ; Christopher M. Bancroft ; Peter F. Bloser ; Jason S. Legere ; Mark L. McConnell and James M. Ryan "Plans for the first balloon flight of the gamma-ray polarimeter experiment (GRAPE)", Proc. SPIE 7732, Space Telescopes and Instrumentation 2010: Ultraviolet to Gamma Ray, 77324E (July 29, 2010); doi:10.1117/12.857467; http://dx.doi.org/10.1117/12.857467

This Conference Proceeding is brought to you for free and open access by the Institute for the Study of Earth, Oceans, and Space (EOS) at University of New Hampshire Scholars' Repository. It has been accepted for inclusion in Space Science Center by an authorized administrator of University of New Hampshire Scholars' Repository. For more information, please contact Scholarly.Communication@unh.edu. 


\section{Authors}

Taylor Connor, Chris Bancroft, Peter F. Bloser, Jason S. Legere, Mark L. McConnell, and James M. Ryan 


\title{
Plans for the First Balloon Flight of the Gamma-Ray Polarimeter Experiment (GRAPE)
}

\author{
Taylor P. Connor, Christopher M. Bancroft, Peter F. Bloser, \\ Jason S. Legere, Mark L. McConnell ${ }^{1}$, and James M. Ryan \\ Space Science Center, University of New Hampshire, Durham, NH 03824
}

\begin{abstract}
We have developed a design for a hard X-ray polarimeter operating in the energy range from 50 to $500 \mathrm{keV}$. This modular design, known as GRAPE (Gamma-Ray Polarimeter Experiment), has been successfully demonstrated in the lab using partially polarized gamma-ray sources and using fully polarized photon beams at Argonne National Laboratory. In June of 2007, a GRAPE engineering model, consisting of a single detector module, was flown on a high altitude balloon flight to further demonstrate the design and to collect background data. We are currently preparing a much larger balloon payload for a flight in the fall of 2011. Using a large (16-element) array of detector modules, this payload is being designed to search for polarization from known point sources of radiation, namely the Crab and Cygnus X-1. This first flight will not only provide a scientific demonstration of the GRAPE design (by measuring polarization from the Crab nebula), it will also lay the foundation for subsequent long duration balloon flights that will be designed for studying polarization from gamma-ray bursts and solar flares. Here we shall present data from calibration of the first flight module detectors, review the latest payload design and update the predicted polarization sensitivity for both the initial continental US balloon flight and the subsequent long-duration balloon flights.
\end{abstract}

Keywords: polarimetry, polarization, gamma-ray, X-ray, balloon, Crab Nebula, gamma-ray burst, solar flare

\section{INTRODUCTION}

GRAPE (Gamma RAy Polarimeter Experiment) is a Compton polarimeter that operates at the hard X-ray/soft gamma-ray energy band (50-500 keV). GRAPE is designed to investigate the acceleration mechanisms of astronomical phenomena, such as gamma-ray bursts (GRBs), solar flares, and pulsars using polarization measurements. In the case of GRBs, for example, polarization measurements could serve to distinguish between two basic models. ${ }^{1}$ We have constructed a prototype GRAPE module that has been calibrated at a polarized hard X-ray beam ${ }^{2}$ and flown on an engineering balloon test flight ${ }^{3}$. Currently, a science capable payload is being constructed and calibrated in preparation for a high altitude balloon flight in the fall of 2011. While GRAPE was designed primarily for un-collimated (wide FoV) observations of bright, short-lived events, like solar flares and GRBs, ${ }^{2-8}$ it will be operated in a collimated mode for this first balloon flight. The primary target of that flight will be the Crab Nebula, with Cygnus X-1 and the Sun (solar flares) as secondary targets. Our observations of the Crab will be compared to the findings of the INTErnational Gamma-Ray Astrophysics Laboratory (INTEGRAL) ${ }^{9,10}$, which provided constraints on the location of the particle acceleration region within the Crab Nebula. Following the initial flight in 2011, the long-range plan is to fly a series of long duration balloon flights from Antarctica to study GRBs and solar flares.

\section{COMPTON POLARIMETRY}

Compton Polarimetry, which uses Compton scattering, is the primary technique used to measure polarization in the $50-500 \mathrm{keV}$ energy band. ${ }^{11}$ When X-ray and gamma-ray photons Compton scatter they tend to scatter with an azimuthal scatter angle that is at a right angle with respect to the incident polarization vector. For unpolarized beams, there is no preferred direction for the azimuthal scatter angle. With a polarized beam, with its net positive electric field vector, there is a preferred azimuthal scatter angle. We exploit this fact to measure the polarization of the incident radiation by looking at the azimuthal distribution of the scattered photons. This distribution gives us a measure of both the level of polarization and the orientation of the polarization vector.

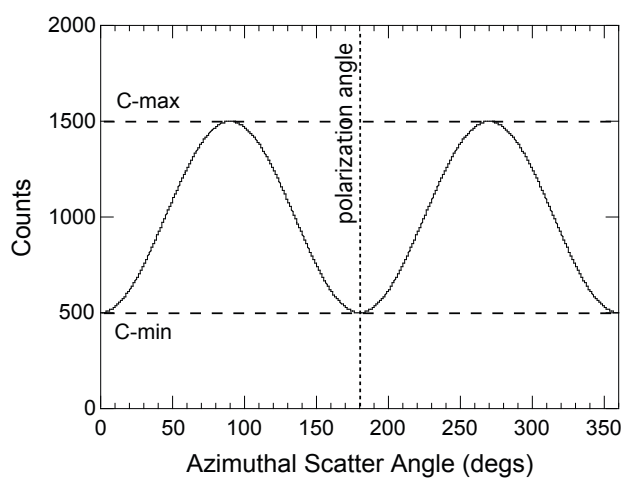

Figure 1: Ideal distribution of azimuthal scatter angles for a polarized photon beam, also referred to as the modulation pattern.

${ }^{1}$ e-mail correspondence: mark.mcconnell@unh.edu 
One can measure a Compton scatter by using two separate detectors. One, the scattering detector, is where the Compton scatter occurs. The other, the calorimeter, is where the remaining photon energy is absorbed. Ideally, the scattering detector is comprised of low $\mathrm{Z}$ material, so Compton scattering is the preferred reaction for the high-energy photon. Also, low $\mathrm{Z}$ material increases the probability that the photon will escape after only one event. The calorimeter should be composed of high $\mathrm{Z}$ material so that the scattered photon can be fully absorbed in a photoelectric event.

The measured location of the two interaction sites (as defined by each of the detectors) is used to determine the azimuthal angle of the scattered photon, and the modulation pattern of the incident flux. The ability to measure the location of each interaction site therefore becomes an important consideration in the design of the detector. The polarization response of a detector is defined in terms of the modulation factor $(\mu)$, which is a measure of the amplitude of the modulation profile resulting from a fully polarized incident beam (Figure 1).

$$
\mu=\frac{C_{\max }-C_{\min }}{C_{\text {max }}+C_{\text {min }}}
$$

The polarization parameters are determined from the measured modulation pattern. The fractional polarization is determined by comparison of the measured modulation factor with that expected for a fully polarized beam. The polarization angle is defined by the minimum in the measured modulation profile. Asymmetries in the instrument response (which can obfuscate the true modulation pattern of the incident) can be dealt with using the instrument response to unpolarized radiation (either measured or simulated). Optionally, instrument systematics can be mitigated by constant rotation of the polarimeter.

The ability of a detector to measure polarization is defined by the so-called minimum detectable polarization (MDP). ${ }^{12}$ This depends not only on the source and background counting rates, but also on the modulation factor:

$$
\operatorname{MDP}(\%)=\frac{n_{\sigma}}{\mu R_{s r c}} \sqrt{\frac{2\left(R_{s r c}+R_{b g d}\right)}{T}}
$$

Here $\mathrm{n}_{\sigma}$ is the significance level (number of sigma), $\mathrm{R}_{\text {src }}$ is the total source counting rate, $\mathrm{R}_{\text {bgd }}$ is the total background counting rate and $\mathrm{T}$ is the total observation time.

\section{THE GRAPE POLARIMETER DESIGN}

The GRAPE design is based on a hybrid assembly of low-Z and high- $Z$ scintillator elements. ${ }^{2-8}$ The scintillator assembly is read out by a multi-anode photomultiplier tube (MAPMT), with each anode providing the readout of a single scintillator element. The arrangement of high- $Z$ and low- $Z$ scintillator elements was determined through simulations. Coincidence events between low- $Z$ and high- $Z$ elements provide a measure if the azimuthal scattering angle of the incident photon.

In order to evaluate the characteristics of the design, an Engineering Model (EM) was developed. 2,3 The geometry of the EM scintillator assembly is shown in Figure 2 . In this arrangement, a single $\operatorname{CsI}(\mathrm{Na})$ element $(10 \mathrm{~mm} \times 10 \mathrm{~mm} \times 50 \mathrm{~mm}$ in size $)$ is surrounded by 60 plastic scintillator elements (each $5 \mathrm{~mm} \times 5 \mathrm{~mm} \times 50 \mathrm{~mm}$ in size). The plastic elements provide the low-Z medium for Compton scattering. The CsI(Na) element provides the high- $Z$ material for photoelectric absorption. Each scintillator element is independently wrapped in reflective VM2000 ${ }^{\mathrm{TM}}$ to improve optical light collection. Readout of the scintillator assembly is provided by a 64 channel Hamamatsu multi-anode photo-multiplier tube (MAPMT model H8500). The MAPMT has an $8 \times 8$ array of $5 \mathrm{~mm}$ anodes (on a pitch of $6.08 \mathrm{~mm}$ ), each with its own separate output. The 64 anode outputs of the MAPMT are fed into four main readout boards with 16 channels each. The boards include an analog processing chain that consists of a charge pre-amplifier, a constant-fraction discriminator, a pulse-shape discriminator for the plastic elements, a Gaussian shaping filter, and a sample-and-hold.

Pulse shape discrimination is used to mitigate the effects of optical crosstalk between anodes. The design utilizes the fact that the plastic pulse has a much faster decay time $(\sim 1.8 \mathrm{~ns})$ than the $\operatorname{CsI}(\mathrm{Na})(\sim 600 \mathrm{~ns})$. The plastic signal is delayed by $\sim 600$ ns and then the calorimeter signal and the delayed plastic signal are fed into a coincidence circuit with a window of $\sim 100 \mathrm{~ns}$. Any signal from a plastic anode caused by cross talk from the $\operatorname{CsI}(\mathrm{Na})$ is delayed beyond the coincidence window. Some level

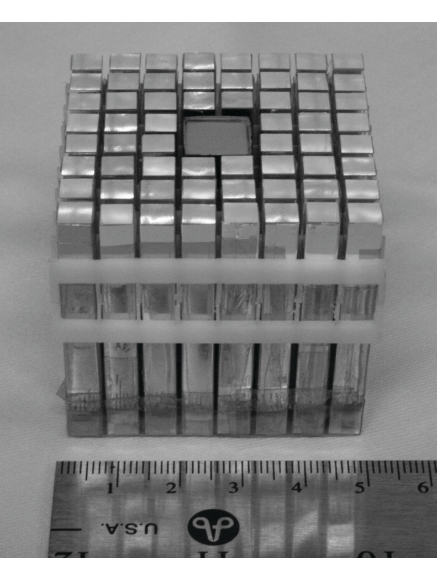

Figure 2: Geometry of the scintillator elements as used in the engineering model, with $1 \mathrm{CsI}(\mathrm{Na})$ scintillator surrounded by 60 plastic scintillators. 
of optical crosstalk is unavoidable while using the H8500 with its 2 mm-thick entrance window, which allows light from each scintillator to reach the neighboring anode(s). This optical crosstalk is in addition to the $3 \%$ intrinsic electronic crosstalk of the H8500 design, as quoted in the technical specifications sheet.

The EM employed compact, low power electronics, custom software and an onboard computer that provided autonomous control and data acquisition (Figure 3). The readout for the EM is controlled by a programable PIC microprocessor. The design was intentionally developed without the use of custom ASICs to assure flexibility with evolving balloon instrument configurations while minimizing the cost of future modifications.

\subsection{Calibration of the Engineering Model}

The module configuration was first tested in the UNH lab using $662 \mathrm{keV}$ photons from a ${ }^{137} \mathrm{Cs}$ source that were scattered in a block of plastic scintillator. ${ }^{5}$ The scattering of $662 \mathrm{keV}$ photons at $90^{\circ}$ creates a beam of partially polarized photons with a polarization of $\sim 55-60 \%$ and a nominal energy of $\sim 288 \mathrm{keV}$. To account for asymmetries within the detector, it was necessary to correct the data collected with a polarized beam using data collected with an unpolarized beam. For the unpolarized data, we used photons from ${ }^{133} \mathrm{Ba}$, which provides $356 \mathrm{keV}$ photons, comparable in energy to the polarized photons created in the lab.

The EM was subsequently calibrated at the Advanced Photon Source (APS) at Argonne National Laboratory in December of $2006 .{ }^{2}$ It was exposed to two linearly polarized beams (at $69.5 \mathrm{keV}$ and $129.5 \mathrm{keV}$ ) both of which were $\sim 97 \%$ polarized. Because the beam was less than $1 \mathrm{~mm}$ in diameter, it was focused at each scintillator element separately. Beam time constraints

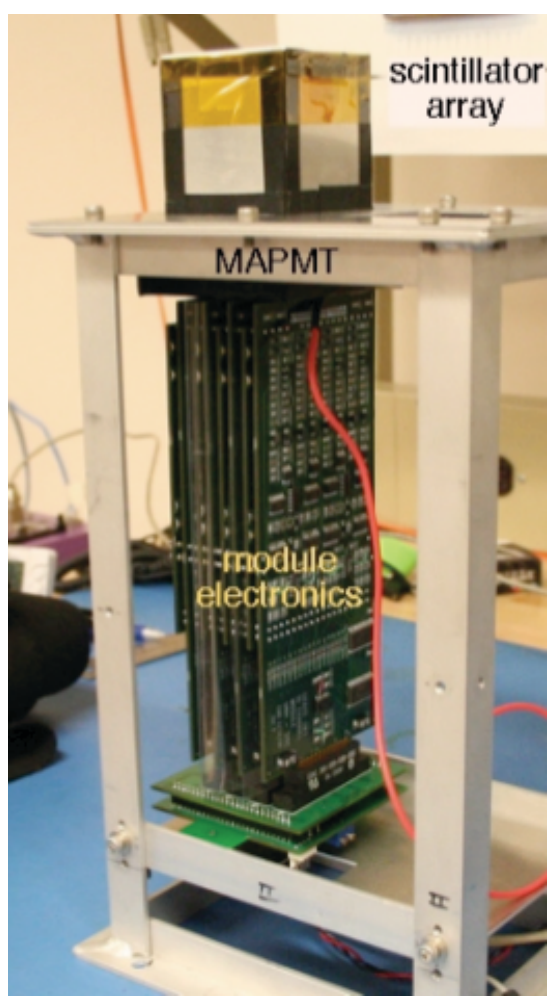

Figure 3: GRAPE Engineering Model (EM), with associated electronics. made it necessary to scan only half of the elements in a checkerboard pattern. The EM was then rotated $90^{\circ}$ and the same elements were scanned again. This effectively provided an unpolarized beam by combining the two sets of data (two sets of data with orthogonal polarizations). Different sets of unpolarized data were used to correct the polarized beam data. The unpolarized data included the combined data runs, laboratory data collected with ${ }^{241} \mathrm{Am}(59.5 \mathrm{keV})$ and ${ }^{57} \mathrm{Co}(122 \mathrm{keV})$, and simulated data based on simulations of the EM configuration. ${ }^{2}$ The resulting polarization signal at $129.5 \mathrm{keV}$ is shown in Figure 4.

\subsection{Engineering Balloon Flight}

The EM was flown on a high-altitude balloon from Palestine, TX in 2007 (Flight 1593-P). ${ }^{3}$ A balloon payload was developed for the EM which included a pressure vessel, supporting electronics and data storage. The module was also surrounded by both passive and active anti-coincidence shielding. The balloon payload remained at float $(\sim 130,000 \mathrm{ft})$ for 5.5 hours before termination of the flight. The EM was retrieved in working condition and the onboard data were recovered. The module was not designed to be sufficiently sensitive to provide any meaningful scientific information, but it did provide valuable engineering data and it did demonstrate the effectiveness of the electronics design. Analysis of the data indicated a stable 50-300 keV background rate for valid events of $\sim 1.1 \mathrm{cts} \mathrm{s}^{-1}$ per module. In this case, valid events are those that trigger one plastic and one calorimeter element and are not vetoed by the active anti-coincidence shielding.

\section{BALLOON PAYLOAD}

With the success of the engineering test fight, a full-scale (science capable) balloon payload has been designed and is

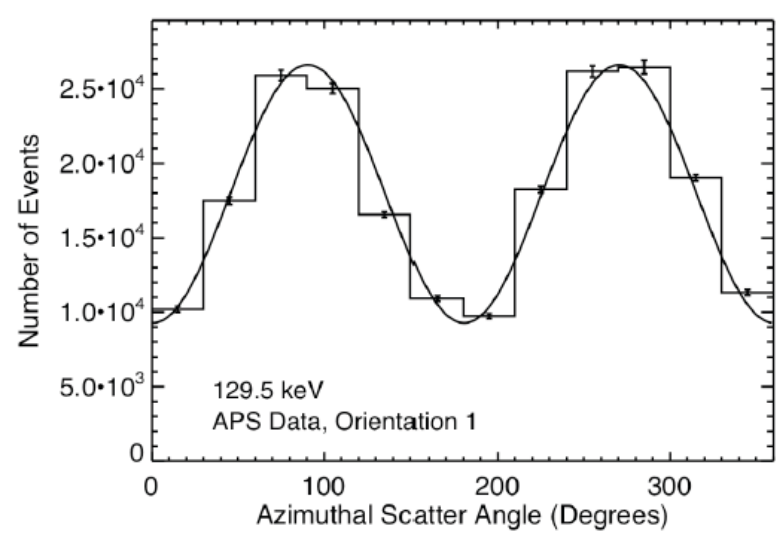

Figure 4: Polarization signature recorded with the EM using a $97 \%$ polarized $129 \mathrm{keV}$ photon beam at Argonne National Laboratory. The measured modulation factor was $\mu \cong$ $0.48 \pm 0.03$. 
being prepared for flight. The first flight of this payload will take place in the fall of 2011 from Ft. Sumner, NM. The flight instrument consists of 16 polarimeter modules arranged in a $4 \times 4$ configuration. The array will be passively collimated with a $\sim 10^{\circ}$ field of view (FoV). The primary target for the first flight will be the Crab. Secondary targets include Cygnus X-1 and the Sun (if it is active).

\subsection{Detector and Instrument Configuration}

Monte Carlo simulations were used to optimize the GRAPE design. The final polarimeter module design is shown in Figure 5. The scintillator arrangement differs from that in the Engineering Module. This rearrangement results from simulation studies and provides a significant increase in effective area. Each module is constructed as a $6 \times 6$ configuration of 36 plastic scintillators $\left(5 \times 5 \times 50 \mathrm{~mm}^{3}\right.$ in size $)$ surrounded by $28 \mathrm{CsI}(\mathrm{Tl})$ scintillators $\left(5 \times 5 \times 50 \mathrm{~mm}^{3}\right.$ in size).

The layout of the detector assembly is an array of 16 polarimeter modules (Figure 6). (In anticipation of future balloon missions, the payload is designed to accommodate up to 32 polarimeter modules.) Each module has four 16channel analog electronics boards to process the MAPMT anode signals. Each channel has a fast discriminator to generate triggers for the hit scintillator elements and a slow shaper with peak-detect-and-hold circuitry that forms part of the pulse height measurement. An interface board and a logic board with fast coincidence circuitry to recognize coincident and anti-coincident events, validate each event, multiplex and digitize the pulse heights and assemble event messages for further processing, complete the module electronics. Module housings are designed so that assembly, test, calibration and debug activities can be conducted on individual modules outside of the detector array.

The anti-coincidence detector is composed of 6 rectangular panels mounted to an aluminum frame to surround the polarimeter array on its four sides, top and bottom. Each panel has a 5-mm thick sheet of plastic scintillator contained in a rigid, light tight (honeycomb) aluminum housing. The design uses a wavelength shifting (WLS) bar and two PMTs (one on each end of the WLS bar) to read out the signal from each scintillator panel. This approach was developed in laboratory studies at UNH for FiberGLAST ${ }^{13}$ and was used for the EM balloon payload. It features easily handled modular panels and generates strong signals with relatively thin scintillator sheets.

A multi-layer passive shield, $\mathrm{Pb}$ and $\mathrm{Cu}$, surrounds the polarimeter detector array on five sides to provide passive shielding. These layers attach to the inner walls of the side and bottom anti-coincidence panels, with the copper facing the detector array. The passive shield serves to absorb atmospheric hard X-ray radiation from below and to the sides of the payload, with the inner, lower-Z layers blocking any fluorescent X-ray emission from the outer, higher-Z layers. Passive multi-layer absorbers, $\mathrm{Pb}$ and $\mathrm{Cu}$, will also be used to collimate the response of the detector array to within an acceptance angle of $\sim 10^{\circ}$. The collimators will extend $\sim 20 \mathrm{~cm}$ in front of the sensitive detector volume, but not between them, and will be composed of $1 \mathrm{~mm}$ of $\mathrm{Pb}$ and $0.5 \mathrm{~mm}$ of $\mathrm{Cu}$ facing each module.

There is provision for handling those events that scatter from a CsI calorimeter element in one module into a CsI calorimeter element in an adjacent module, since simulations show that there are a significant number of such events (section 5).

\subsection{Payload Configuration}

The balloon gondola for the Ft. Sumner flight is illustrated in Figure 7. The instrument components are mounted inside a pressure vessel. An axial rotation mechanism, designed to mitigate the effects of instrumental systematics by rotating the instrument back and forth $\left( \pm 90^{\circ}\right)$ about the pointing direction, is also mounted inside the pressure vessel. An external frame (fabricated from extruded aluminum stock) supports and protects the instrument pressure vessel and provides the mounting structure for the remaining UNH and CSBF payload components. The pressure vessel is a three-piece aluminum assembly consisting of a cylindrical sidewall and upper and lower domes. It will contain one atmosphere (15 psia) of gaseous nitrogen (GN2). All instrument hardware is supported from a base plate mounted to the sidewall. The detector modules, six anticoincidence panels and the Module Interface Board (or MIB, which provides electronic and mechanical mounting for each polarimeter

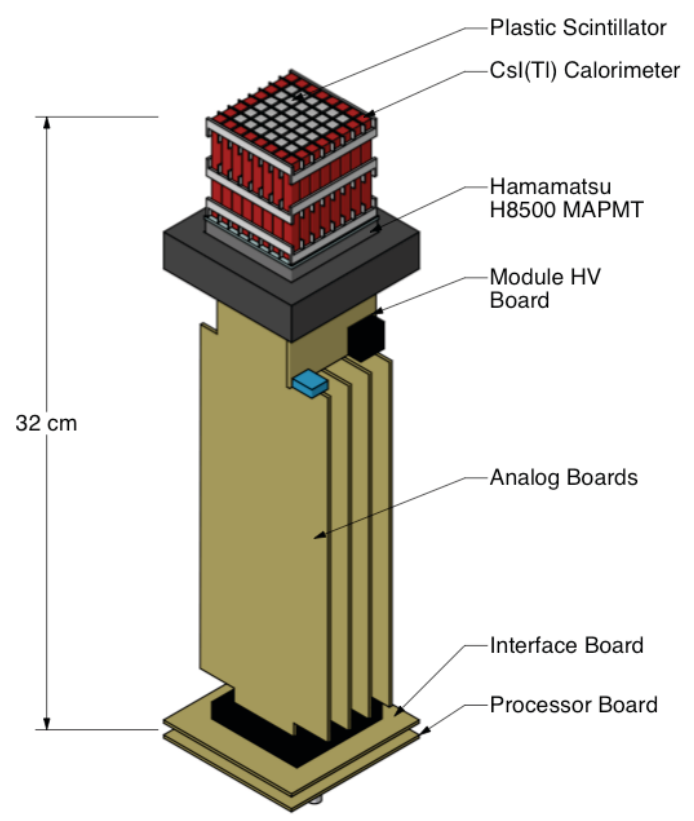

Figure 5: Schematic of the flight configuration of a single polarimeter module, showing the various electronics boards. 
module) mount to the top of this base plate; the bottom anti-coincidence panel, the instrument computer and the instrument power supply and distribution module mount to the bottom side of this base plate. The sidewall has ports for electrical and gas feed-throughs and brackets for secure mounting to the frame. The pressure vessel's top and bottom dome elements are formed with aluminum. The thickness of the top dome will kept as small as possible to allow for photon transmission. The temperature within the pressure vessel will be maintained in the $-10^{\circ}$ to $+30^{\circ} \mathrm{C}$ range using fans and heaters.

The requirement of the attitude control system (ACS) will be to maintain the source target within the $\sim 10^{\circ} \mathrm{FoV}$. Control precision of $\pm 3^{\circ}$ is adequate with pointing knowledge uncertainty of $\pm 0.5^{\circ}$. A reaction wheel and momentum transfer unit and an elevation drive mechanism will be employed. A commercial differential GPS unit will be used to provide the gondola's 3-dimensional orientation at frequent intervals. A magnetometer-based digital compass and GPS sensor will act as a backup. The elevation angle will be measured with encoders and recorded along with the time-tagged azimuth information.

An effective way to deal with the problem of instrumental systematics is to induce a rotation of the polarimeter about the pointing axis. Rather than having a continuous $360^{\circ}$ rotation, the payload will employ a simpler (and, for our purposes, equivalent) design that rotates the detector back and forth through a range of $180^{\circ}\left( \pm 90^{\circ}\right)$. A drive for axial rotation of the instrument will be implemented within the pressure vessel to provide a rotation rate of $\sim 180^{\circ}$ per minute. An encoder in the axial drive system will measure and record the time-tagged orientation as part of the housekeeping data; modest knowledge of this orientation $\left( \pm 1^{\circ}\right)$ is adequate.

\section{SIMULATIONS}

In order to be useful for polarization studies, an event must include two (and only two) interaction sites. The two interaction sites define an azimuthal scatter angle under the assumption that the incident photon undergoes a Compton scatter in one interaction and a photoelectric absorption in the second interaction. Because of the $180^{\circ}$ symmetry of the Compton scatter process, it is not necessary to know which inaction takes place first. We can define three different types of valid twosite events; 1) plastic-calorimeter (P-C) events;

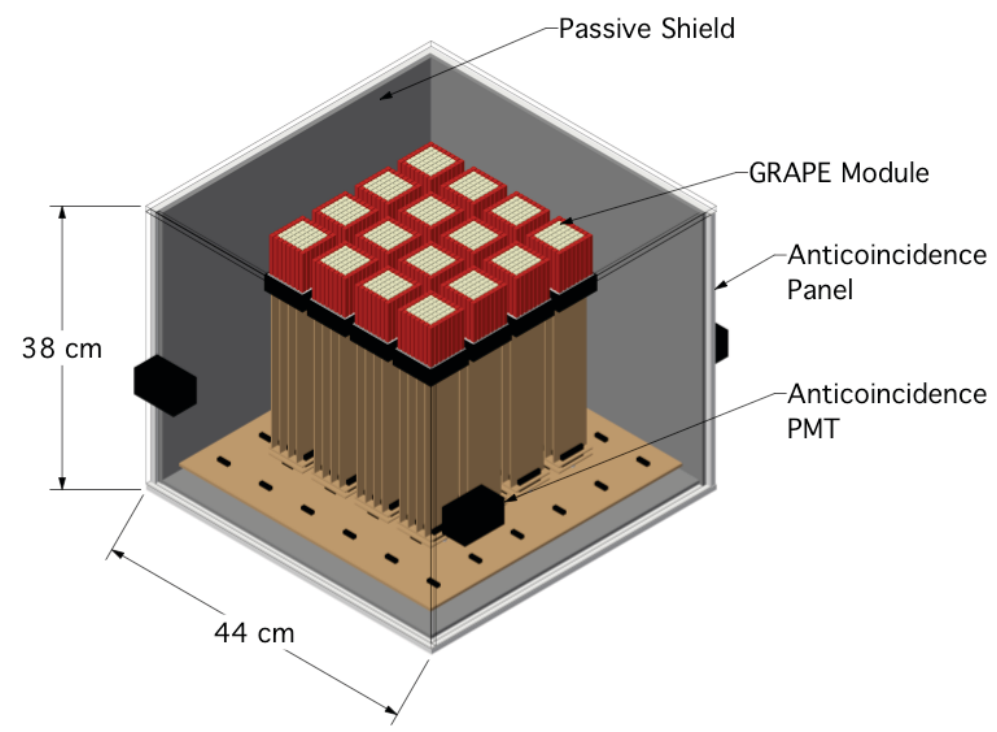

Figure 6: The GRAPE instrument assembly with the $4 \times 4$ module array. The design can accommodate up to 32 polarimeter modules. Only 16 modules will be flown on the first flight. The individual module housings and the collimator assembly are not shown.

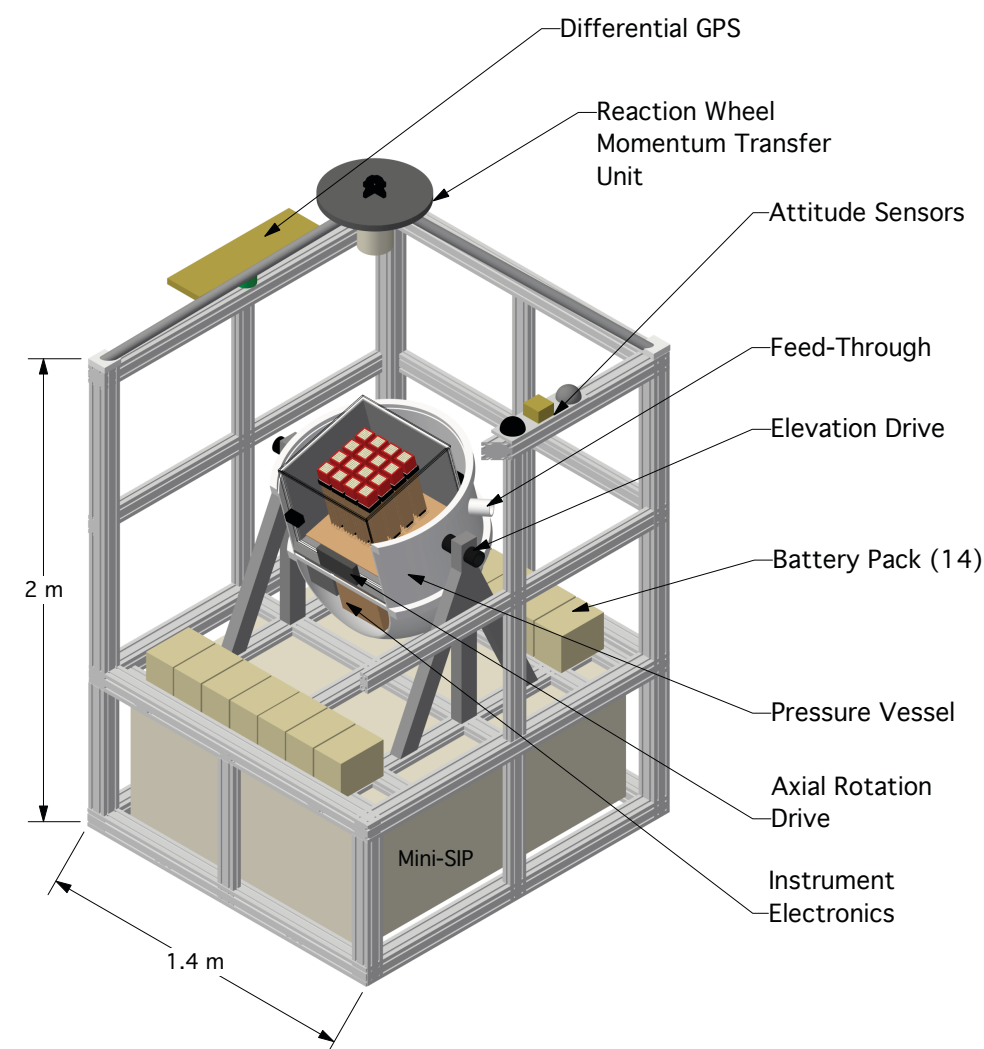

Figure 7: The balloon gondola, shown with in $4 \times 4$ module configuration. The entire design (both mechanical and electrical) can accommodate up to 32 polarimeter modules in anticipation of future long duration balloon flights. 

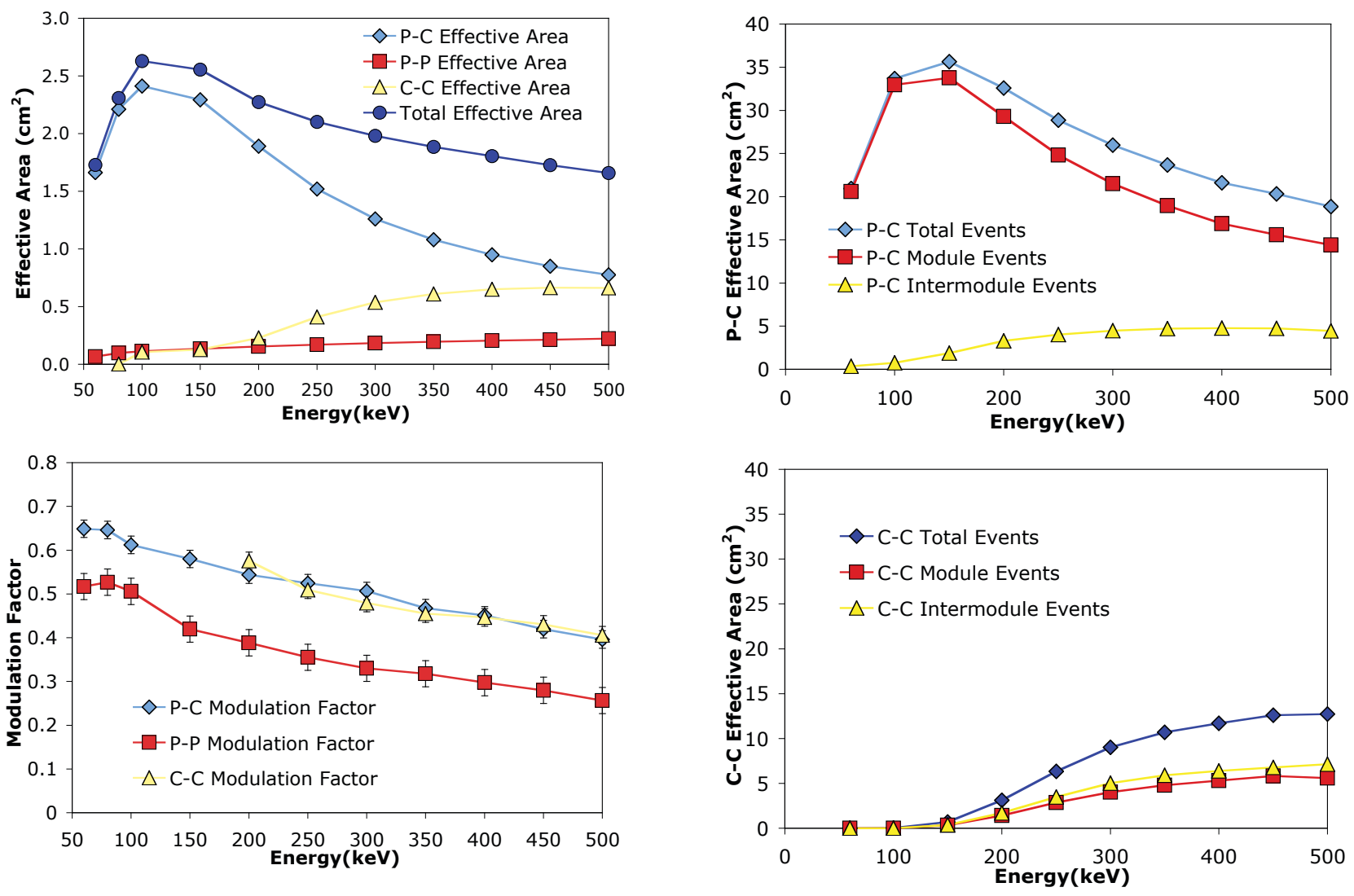

Figure 8: Simulated response parameters for a single polarimeter module. Shown here are the on-axis detection efficiency (top) and the modulation factor for a fully polarized beam (bottom) vs. energy. There are too few C-C events at energies below $200 \mathrm{keV}$ to derive a modulation factor.

2) plastic-plastic (P-P) events; and 3) calorimetercalorimeter (C-C) events. Simulations (based on GEANT4) have been used to study these events in a single polarimeter module and in a 16-element $(4 \times 4)$ module array. Simulations scanned an energy range from 50 to $500 \mathrm{keV}$.

The single module simulations included only a bare scintillator assembly (plastic and CsI elements). The simulation results are shown in Figure 8. The effective area is shown for each of the three valid event types. The P-C events were the most common at all energies, and thus give the highest effective area. The C-C events contribute to the effective area only above $200 \mathrm{keV}$. The P-P events always contribute at a much lower level with respect to the other

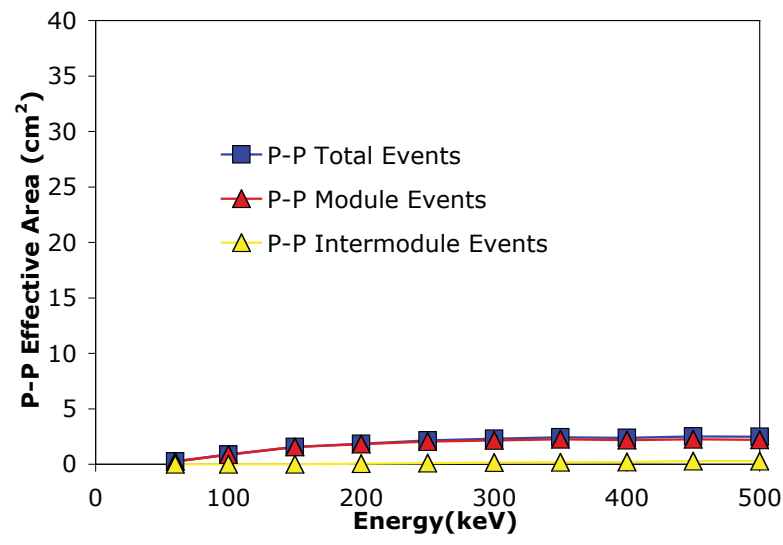

Figure 9: Simulated response a $4 \times 4$ array of polarimeter modules. Shown here are the effective area for each of the various event types (see text), which includes events involving more than one polarimeter module. event types. The modulation factors for the P-C events and $\mathrm{C}-\mathrm{C}$ events are comparable at all energies where the $\mathrm{C}-\mathrm{C}$ events contribute. The modulation factors for the P-P events are consistently lower, presumably due to the shorter path-length between interaction sites, which leads to larger uncertainties in the azimuthal scatter angle.

The module array simulations included a more realistic mass distribution, one that approximates the mass distribution in the payload design. Each individual module includes a $1 \mathrm{~mm}$ Al housing around the scintillator assembly. The entire array is surrounded by $6 \mathrm{~mm}$ of plastic anti-coincidence shielding and a multi-layer passive shield consisting 
of $\mathrm{Cu}-\mathrm{Pb}-\mathrm{Sn}$. The collimator assembly, designed to collimate each individual module, extends $20 \mathrm{~cm}$ in front of the scintillators (but not between the modules) and consists of a $1 \mathrm{~mm}$ layer of $\mathrm{Pb}$ with $0.5 \mathrm{~mm}$ of $\mathrm{Cu}$ facing each module. Some allowance is also made for the mass of the PMTs and associated electronics. The entire instrument assembly is then surrounded by an $\mathrm{Al}$ pressure vessel that is $3 \mathrm{~mm}$ thick.

The results for the array simulations are shown in Figure 9. For these simulations, two-site events involving more than one module were also considered. These events can contribute significantly to the total response of the array, especially at higher energies. Events contained within a single module are referred to as "module events." Events that involve two different modules (with one interaction in each module) are referred to as "intermodule events." In all cases, the P-P events (both module and intermodule events) do not contribute significantly to the instrument response. In the case of intermodule P-P events, this results from the shielding effects of the CsI that surrounds the central plastic elements in each module.

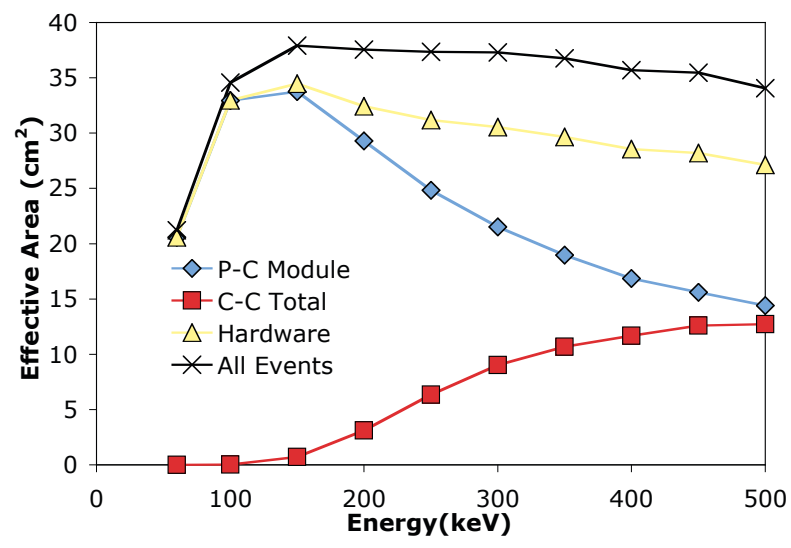

Figure 10: Simulated response a $4 \times 4$ array of polarimeter modules. Shown here is the effective area for P-C module events, $\mathrm{C}-\mathrm{C}$ events (both module and intermodule combined) and the combination of those two (labeled "hardware" to reflect that the GRAPE hardware processes only these events). Also shown here is the total effective area for all valid event types, some of which are not processed in hardware.

The GRAPE hardware is designed to process only the following types of valid events : P-C module events, C-C module events and C-C intermodule events. Simulations have shown that these are the dominant event types. The effort required to develop the complex hardware required to process other event types is not justified at this time. The total effective area for these event types only is shown in Figure 10 and labeled as "hardware" to indicate that these are the events handled by the GRAPE hardware. For comparison, the total effective area for all valid event types is also shown.

\section{FLIGHT PLAN}

For the first balloon flight, GRAPE will be configured as a $4 \times 4$ module array, with collimation of each module providing a $10^{\circ} \mathrm{FoV}$. The average counting rate from the Crab was estimated using simulations based on an atmospheric depth of $3.5 \mathrm{~g} \mathrm{~cm}^{-2}$ and a total observation time of 8 hours at an average Crab zenith angle of $30^{\circ}$ (typical numbers for the latitude of Ft. Sumner). The background was extrapolated from that measured during the 2007 balloon flight. Only valid $\mathrm{P}-\mathrm{C}$ events within each module were considered. We estimate a minimum detectable polarization ( $3 \sigma \mathrm{MDP})$ level of $\sim 13 \%$ for the Crab in the $50-500 \mathrm{keV}$ energy range. (We are currently working to update the sensitivity estimates to include C-C events, which are expected to improve these numbers.) Measurements of the Crab nebula polarization with instruments on the INTEGRAL spacecraft indicate polarization levels in excess of $40 \%$ at GRAPE energies. ${ }^{9,10}$ These results indicate that GRAPE should be able to measure the polarization of the Crab nebula during a single source transit. Observations of Cygnus X-1, with an expected polarization level of less than a few percent, will provide a good null test for GRAPE. Observations of both sources will be possible for a 24-hour balloon flight, with two transits of the Crab for a 36-hour flight.

\section{SUMMARY}

Currently, the first flight module for the 2011 balloon flight has been assembled (Figure 11) and is currently being calibrated. The calibration data will be used to provide energy calibrations, to characterize the optical cross talk between

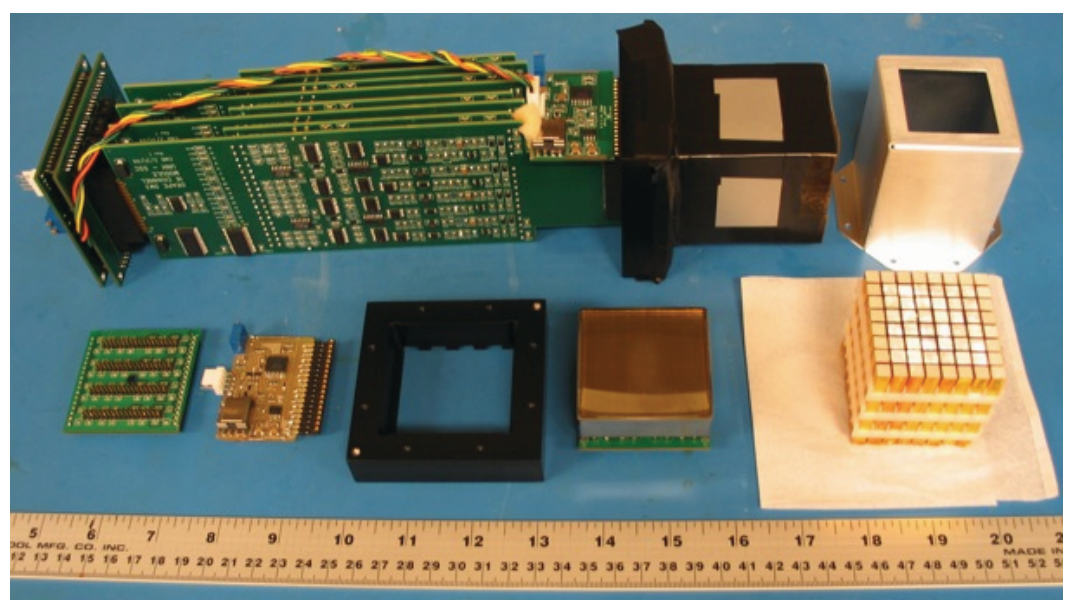

Figure 11: The first assembled flight module (top), shown here with some of the individual module components. 
elements and to calibrate the polarization response. The polarization calibration procedure is similar to that used for the EM (section 3.1), but will employ a continuous rotation of the polarimeter module as a means to remove systematic effects in the measurement. This will mimic the approach that will be employed for the balloon payload.

The long range program goal for GRAPE is to fly a 32 module array on a series of Long Duration Balloon (LDB) missions. These LDB flights will last for 20-30 days and will be conducted from the McMurdo station in Antarctica. The goal of these flights would be to provide polarization measurements of gamma-ray bursts and solar flares. To maximize sky exposure to randomly located GRBs, the polarimeter array will be uncollimated with a $\sim 120^{\circ} \mathrm{FoV} .^{7,8}$ To maximize the chances of making solar flare measurements, we hope to have our first LDB flight at the end of 2012.

\section{REFERENCES}

[1] Toma, K., et al., "Statistical Properties of Gamma-Ray Burst Polarization,” Ap. J. 698, 1042-1053 (2009).

[2] Bloser, P. F., et al., "Calibration of the Gamma-RAy Polarimeter Experiment (GRAPE) at a polarized hard X-ray beam,” Nuc. Instr. Meth. A 600, 424-433 (2009).

[3] McConnell, M.L., et al., “GRAPE : A balloon-borne gamma-ray polarimeter," Proc. SPIE 7435, 74350J (2009).

[4] McConnell, M.L., et al., "Dedicated polarimeter design for hard x-ray and soft gamma-ray astronomy," Proc. SPIE $5165,334-345$ (2004).

[5] Legere, J., et al., "Developing a Compton polarimeter to measure polarization of hard x-rays in the 50-300 keV energy range," Proc. SPIE 5898, 413-422 (2005).

[6] Bloser, P.F., et al., "GRAPE - A Balloon-Borne Gamma-Ray Polarimeter Experiment," Chinese J. Astron. Ast. Supp. 6, 393-397 (2006).

[7] McConnell, M.L., et al. "Prospects for GRB Polarimetry with GRAPE,” AIP Conf. Proc. 836, 654-659 (2006).

[8] McConnell, M.L., et al., “A Balloon-Borne Gamma-Ray Polarimeter for Gamma-Ray Bursts,” AIP Conf. Proc. 1133, 61-63 (2009).

[9] Dean, A.J., et al., "Polarized Gamma-Ray Emission from the Crab," Science 321(5893), 1183-1185 (2008).

[10] Forot, M., et al., "Polarization of the Crab Pulsar and Nebula as Observed by the INTEGRAL/IBIS Telescope," Ap. J. 688, L29-L32 (2008).

[11] Lei, F., A.J. Dean, and G.L. Hills, “Compton Polarimetry in Gamma-Ray Astronomy,” Space Sci. Rev. 82, 309-388 (1997).

[12] Novick, R., "Stellar and Solar X-Ray Polarimetry," Space Sci. Rev. 18, 389-408 (1975).

[13] Pendleton, G.N., et al. "FiberGLAST: a scintillating fiber approach to the GLAST mission," Proc. SPIE 3765, 12-21 (1999). 